\title{
Morbus Morbihan
}

\section{G. Wagner \\ C. Diaz $^{2}$}

\section{Morbihan's Disease}

\section{Zusammenfassung}

Der Morbus Morbihan, der sich klinisch durch persistierende Erytheme und Schwellungen des Gesichtes auszeichnet, ist eine selten beschriebene Dermatose ungeklärter Ätiologie und Pathogenese. Bisweilen werden Zusammenhänge mit der Rosazea oder der Acne vulgaris diskutiert. Bei der hier vorgestellten Patientin entwickelte sich der Morbus Morbihan wenige Wochen nach Katarakt-Operationen beider Augen.
Abstract

Morbihan's disease is a rare dermatosis characterized by persisting facial erythema and oedema of unknown aetiology und pathogenesis. A correlation to rosacea and acne vulgaris has been previously discussed. In the presented case Morbihan's disease developed some weeks after cataract surgeries on both eyes.

\section{Einleitung}

Der Morbus Morbihan, der erstmals 1957 von Degos beschrieben wurde, ist gekennzeichnet durch chronisch-persistierende Erytheme und Schwellungen des Gesichtes, die bevorzugt im Bereich der Stirn, am Nasenrücken, an den Lidern und den Wangen auftreten [1]. In extrafazialer Lokalisation ist der Morbus Morbihan bisher nicht beschrieben worden. Für den klinischen Befund typisch sind anfänglich noch rückbildungsfähige, später jedoch persistierende weinrote bis livide Erytheme in Verbindung mit polsterartigen, im weiteren Verlauf zunehmend derben Schwellungen, die monströse, die ursprünglichen Gesichtskonturen entstellende Ausmaße annehmen können. Ein Ödem im klinischen Sinne einer flüchtigen Schwellung liegt nicht vor, so dass die Begriffe Pseudoödem, Induration oder teigige Schwellung für diese eigentümliche klinische Manifestation vorgeschlagen worden sind [2]. Pruritus, Schmerzen oder andere Formen subjektiver Missempfindungen werden von den betroffenen Patienten im Allgemeinen verneint.
Fehlende laborchemische Veränderungen, unspezifische histopathologische Befunde und eine ungewöhnliche Therapieresistenz zählen hingegen zu den weiteren Merkmalen des Morbus Morbihan.

\section{Kasuistik}

\section{Anamnese}

Die heute 79-jährige Patientin musste sich jeweils im Juli und August 2001 einer Katarakt-Extraktion beider Augen unterziehen, wobei die Patientin gleichzeitig mit Kunststofflinsen versorgt worden war. Im Oktober des Jahres kam es erstmals zu nicht juckenden, anfänglich noch rückbildungsfähigen, seit Januar 2002 dann jedoch persistierenden Rötungen und Schwellungen im Bereich des Gesichtes. Die im Zusammenhang mit den Katarakt-Operationen und mit später auch hinzutretenden Blepharitiden notwendig gewordene ophthalmologische Lokaltherapie wurde mehrmals verändert, ohne dass diese Umstellungen

${ }^{2}$ Einsendungslabor für Dermatopathologie Freiburg

Korrespondenzadresse

Dr. G. Wagner · Hautklinik · Zentralkrankenhaus Reinkenheide · Postbrookstr. $103 \cdot 27574$ Bremerhaven

Bibliografie

Akt Dermatol 2003; 29: 300-303 @ Georg Thieme Verlag Stuttgart · New York · ISSN 0340-2541 


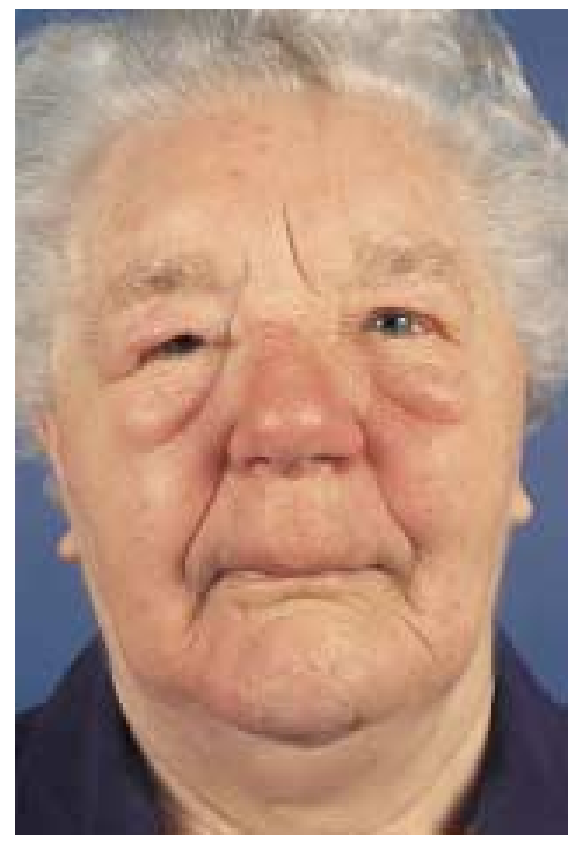

Abb. 1 Morbus Morbihan: Persistierende Erytheme und Schwellungen.

einen feststellbaren Einfluss auf die Rötungen und Schwellungen des Gesichtes zeigten. Auch die seit dem Sommer 2002 erfolgte fachdermatologische Behandlung mit topischen Glukokortikoiden, Erythromycin- oder Metronidazol-Rezepturen sowie die orale Verordnung von Glukokortikoiden oder verschiedenen Antihistaminika führte zu keiner Rückbildung der dermatologischen Symptomatik. Im Januar 2003 wurde die Patientin dann erstmals in der Hautklinik Bremerhaven vorgestellt.

\section{Dermatologischer Befund}

Zentrofazial unter Betonung der Augenlider und des Nasenrückens symmetrisch angeordnete, umschrieben erythematöse Schwellungen von teigig-derber Konsistenz. Keine Papeln, Pusteln oder Schuppung nachweisbar (Abb.1).

\section{Befunde diagnostischer Untersuchungen Histopathologischer Befund}

Regelrechte Epidermis. Dilatierte Follikel mit Nachweis von Demodex folliculorum in den Ostia. Mäßig dichtes perivaskuläres und perifollikuläres lymphohistiozytäres Infiltrat. Starkes Ödem der Dermis mit Nachweis von Mastzellen, keine epitheloidzelligen Granulome. Angiektasien in der gesamten Dermis (Abb. 2 u.3)

\section{Allergologische Diagnostik}

In den umfangreichen Epikutantestungen mit den Allergenen Standardreihe, Salbengrundlagen/Emulgatoren, Konservierungsmittel, Desinfektionsmittel, Antioxidantien, Arzneistoffe mit Augenexterna (Fa. Hermal) zeigten sich keine positiven Reaktionen. Auch die Epikutantestungen mit den von der Patientin verwendeten Ophthalmika ergaben keinen Hinweis für eine epidermale Sensibilisierung (Ultracortenol ${ }^{\circledR}$ Augensalbe; Totocortin ${ }^{\circledR}$, Lacophtal ${ }^{\circledR}$ und Indocolir ${ }^{\circledR}$ Augentropfen).

Die Pricktestungen mit Inhalationsallergenen und Lebensmitteln (Fa. Smith Kline Beecham) waren ebenfalls unauffällig.

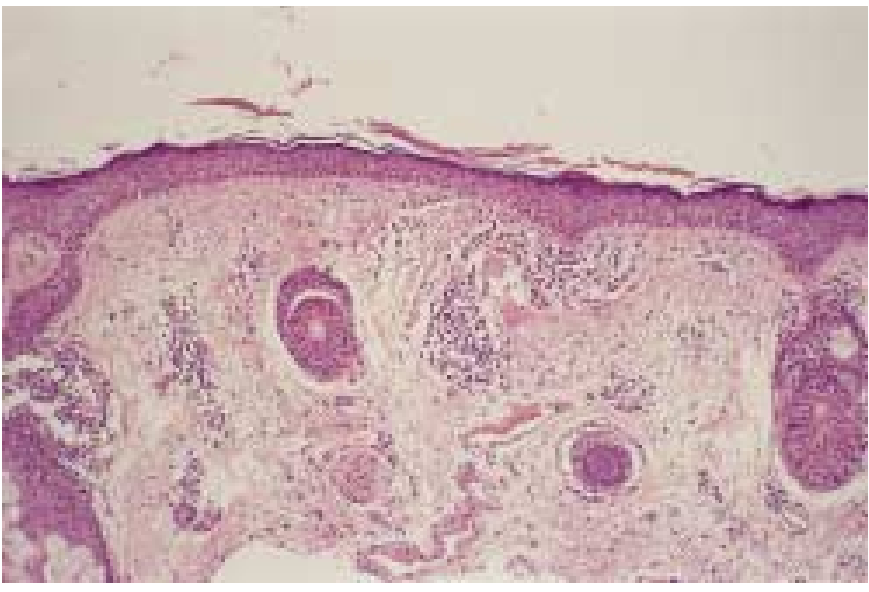

Abb. 2 Perivaskuläres und periadnexielles lymphohistiozytäres Infiltrat, Ödem im Bereich der Dermis (HE × 10).

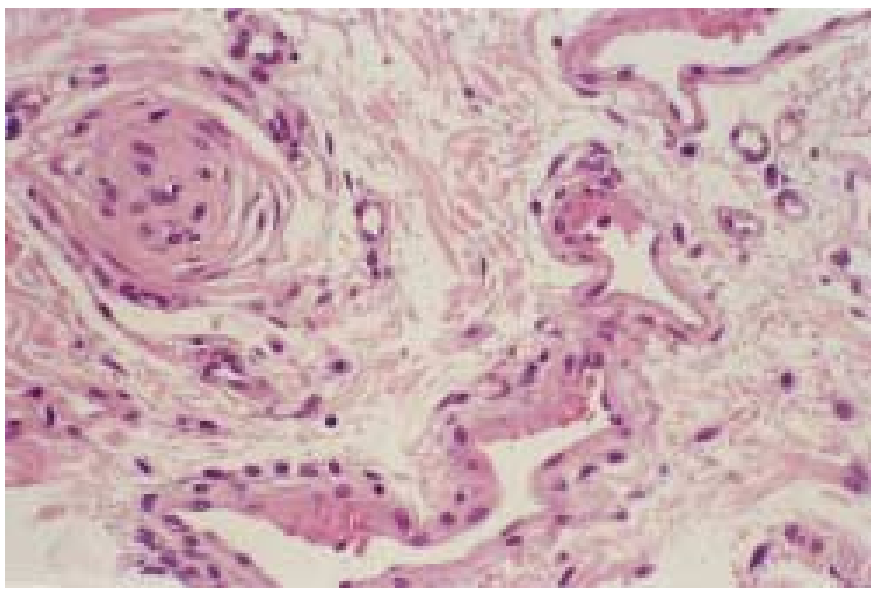

Abb. 3 Auseinander liegende Kollagenbündel als Folge des Ödems, Gefäßektasien (HE × 40). Perivaskuläre Zellen zeigen sich in anderen Färbungen überwiegend als Mastzellen.

\section{Erythemschwellenbestimmung}

Die MDE-Bestimmungen für UVA und UVB lagen mit 25 Joule/ $\mathrm{cm}^{2}$ bzw. mit 0,04 Joule $/ \mathrm{cm}^{2}$ in den entsprechenden Normbereichen.

\section{Laborbefunde}

Keine pathologischen Befunde zeigten sich bei der Bestimmung der üblichen Routineparameter (BB und Diff.-BB, BSG, CRP, Transaminasen, nierenpflichtige Substanzen, Eiweißelektrophorese und Rheumafaktoren). Gesamt-IgE, $C_{1}$-Esterase-Inhibitor und $C_{1}$-Esterase-Inhibitor-Aktivität in den jeweiligen Normbereichen. Antinukleäre Antikörper, antimikrosomale Antikörper und Borrelien-Antikörper nicht nachweisbar. Laborchemisch euthyreote Stoffwechsellage.

\section{Mikrobiologische Untersuchungen}

Im bakteriologischen Abstrich erfolgte der Nachweis von Staphylokokkus aureus. Die mykologischen Untersuchungen waren unauffällig, hierbei insbesondere kein Nachweis von Pityrosporum ovale. 


\section{Ergänzende Untersuchungen}

EKG, Rö.-Thorax, Rö.-NNH und CT-Hirnschädel altersentsprechende, unauffällige Befunde. HNO-, MKG- und Neurol.-Konsil ohne Nachweis entsprechender Grunderkrankungen.

Augenärztliches Konsil: Geringfügige Blepharitis beidseits, glatte unauffällige Kunststofflinsen, unauffällige Schleimhäute. Abheilung der Blepharitis unter Anwendung von Terracortril ${ }^{\circledR}$ Augensalbe.

\section{Therapie und Verlauf}

In Ergänzung zu den bereits erfolgten Behandlungsmaßnahmen wurde gemäß Antibiogramm des nachgewiesenen Staphylokokken-Stammes eine orale Therapie mit Doxycyclin $100 \mathrm{mg} / \mathrm{tgl}$. eingeleitet. Zusätzlich wurde die Patientin lokal mit einer 4\%igen Erythromycin-Rezeptur und im Hinblick auf die histopathologisch nachgewiesenen Demodex-Milben auch mit einer Crotamiton Salbe (Crotamitex ${ }^{\circledR}$ Salbe) behandelt. Eine klinisch feststellbare Verbesserung des dermatologischen Befundes konnte unter dieser Therapie nicht beobachtet werden.

\section{Diskussion}

Im Jahre 1957 beschrieb Degos persistierende Erytheme und Schwellungen des Gesichtes, die in ihrer Ätiopathogenese nicht eingeordnet werden konnten. In Anlehnung an die französische Landschaft Morbihan - hier war der erste Patient beobachtet worden - wurde das Krankheitsbild später als Morbus Morbihan bezeichnet $[1,3]$. Eine vergleichbare klinische Symptomatik ist unter den Diagnosen solides persistierendes Gesichtsödem oder chronisches Ödem und Erythem des Gesichtes bei Patienten mit Acne vulgaris oder Rosazea beschrieben worden [4-6]. Offensichtlich handelt es sich um identische Krankheitsbilder, so dass der Morbus Morbihan einerseits als Komplikation der genannten Dermatosen auftreten kann, andererseits aber auch per se, ohne dass eine vorausgehende oder gleichzeitig bestehende Dermatose beobachtet werden kann, wie dies auch bei der hier vorgestellten Patientin der Fall gewesen ist [7].

Der Morbus Morbihan ist eine seltene, in der Literatur überwiegend als Kasuistik beschriebene Dermatose [1-9]. Obwohl die Morphologie der zentrofazial auftretenden Erytheme und Schwellungen, die Persistenz dieser Symptome und deren Therapieresistenz in ihrer Kombination eigentlich unverwechselbar sein dürften, müssen zahlreiche Differenzialdiagnosen berücksichtigt werden. So ist der Morbus Morbihan trotz seiner charakteristischen Symptomatik in den meisten Fällen als Ausschlussdiagnose angesehen worden, was auch in der umfangreichen Diagnostik der publizierten Kasuistiken zum Ausdruck kommt. Prima vista sind das Quincke-Ödem, die chronische Kontaktdermatitis oder auch ein Erysipel in Betracht zu ziehen. Die bei diesen Diagnosen jedoch fehlende Persistenz der jeweiligen Symptomatik, die bei Quincke-Ödemen häufig vorkommende Beteiligung der Schleimhäute, die in Abhängigkeit vom Verlauf einer Kontaktdermatitis vorhandene Polymorphie mit Bläschen, Schuppung oder Lichenifikationen sowie die beim Erysipel typische Paraklinik mit schwerem Krankheitsgefühl, hohen Temperaturen und Schüttelfrost erlauben in der Regel bereits klinisch eine sichere diagnostische Einordnung der genannten Dermatosen. Auch die für den Morbus Morbihan typische Therapieresistenz findet sich bei diesen Differenzialdiagnosen nicht, da zu deren Behandlung adäquate Verfahren zur Verfügung stehen. Klinischmorphologisch problematischer, histopathologisch jedoch zweifelsfrei zu diagnostizieren sind zahlreiche weitere Dermatosen, die ebenfalls vom Morbus Morbihan abgegrenzt werden müssen. Hierzu zählen so unterschiedliche Erkrankungen wie z.B. das Melkersson-Rosenthal-Syndrom, das Blepharo- oder Metophym, die chronische aktinische Dermatitis, Speicherdermatosen, Lymphome, Kollagenosen und kutane Metastasierungen bei viszeralen Neoplasien $[2,10]$.

Die Histopathologie des Morbus Morbihan selbst ist unspezifisch und zeigt sich in Form einer angiofibromatösen Entzündungsreaktion mit Ödembildung, unterschiedlich ausgeprägter Fibrose und einem vorwiegend perivaskulären, lymphohistiozytären Infiltrat [2]. Auffällig ist eine vereinzelt beschriebene Vermehrung von Mastzellen [11 - 13]. Ein entsprechender Befund konnte auch bei der hier vorgestellten Patientin beobachtet werden. Ob der Vermehrung der Mastzellen eine pathogenetische Bedeutung zugeschrieben werden kann, ist zur Zeit noch nicht geklärt. Einzelne Autoren sehen in einer von den Mastzellen induzierten Fibrose die mögliche Ursache der klinischen Symptomatik des Morbus Morbihan [2,12]. Ein zweiter histopathologischer Befund, der im Zusammenhang mit der Pathogenese des Morbus Morbihan diskutiert wurde, ist die ebenfalls nur gelegentlich beschriebene Besiedlung der Talgdrüsenfollikel mit Demodex folliculorum [2,7]. Bei unserer Patientin hat die lokale Behandlung mit Crotamiton bei histopathologischem Nachweis von Demodex-Milben allerdings keinen Einfluss auf das klinische Bild gezeigt. Weitere mögliche Faktoren, die für die Pathogenese der Erkrankung von Bedeutung sein könnten, sind Traumatisierungen des Gewebes z. B. bei der Therapie der Acne vulgaris oder Störungen der Mikrozirkulation, wie sie auch bei der Rosazea vermutet werden $[4,7,9]$.

Bei der hier vorgestellten Patientin entwickelte sich der Morbus Morbihan in einem unmittelbaren zeitlichen Zusammenhang mit operativen Eingriffen an den Augen, was spekulativerweise mit einer stärkeren Traumatisierung des Gewebes und einer damit verbundenen Störung der Mikrozirkulation verbunden gewesen sein könnte. Allerdings fanden sich in der uns zugänglichen Literatur keine Hinweise für einen postoperativ aufgetretenen Morbus Morbihan. Eine Unverträglichkeitsreaktion auf das Material der implantierten Kunststofflinsen als Ursache des Morbus Morbihan ist bei unserer Patientin so gut wie ausgeschlossen, da der ophthalmologische Befund völlig unauffällig gewesen ist. Darüber hinaus sind auch in der Literatur entsprechende pathogenetische Zusammenhänge bisher nicht beschrieben worden.

Ein weiteres Problem des Morbus Morbihan ist seine ungewöhnliche Therapieresistenz. Dabei sind sehr unterschiedliche Therapieverfahren eingesetzt worden, ohne dass bisher eine Erfolg versprechende Behandlungsmethode entwickelt werden konnte. So erwies sich, von Ausnahmen abgesehen, der Einsatz von Antibiotika als wenig erfolgreich [7-9]. Als vorübergehend wirksam zeigte sich die Therapie mit Prednisolon, wobei diese Behand- 
lung jedoch mit einer täglichen Dosierung von $0,5-1,0 \mathrm{mg} / \mathrm{kg}$ Körpergewicht über mehrere Wochen durchgeführt werden musste [2]. Darüber hinaus wurden vereinzelt Verbesserungen der klinischen Symptomatik während einer Behandlung mit Clofazimin oder Thalidomid beobachtet $[1,14]$. Ausgehend von der These einer möglicherweise vorliegenden Mastzellen induzierten Fibrose wurde von verschiedenen Autoren die Behandlung des Morbus Morbihan mit 0,2-0,7 mg/kg Körpergewicht Isotretinoin in Kombination mit 1-2 mg Ketotifen jeweils tgl. empfohlen. Bei Behandlungszeiten von 4-5 Monaten konnten bei einzelnen Patienten deutliche Rückbildungen der Schwellungen festgestellt werden $[2,13]$. Ein entsprechender Behandlungsversuch wurde von unserer Patientin abgelehnt, so dass bei ihr letztendlich keine Verbesserung des klinischen Befundes erreicht werden konnte.

\section{Literatur}

${ }^{1}$ Laugier P, Gilardi S. L 'oedéme érythémateux chronique facial supérieur (Degos). Ann Dermatol Venereol 1981; 108: 507-513

2 Jansen Th, Plewig G. Morbus Morbihan. Akt Dermatol 1996; 22: $161-164$

${ }^{3}$ Leigheb G, Boggio P, Gattoni M, Bornacina G. A case of Morbihan's disease. Chronic upper facial erythematous oedema. Acta Dermatovenereol A.P.A 1993; 2: 57-61
${ }^{4}$ Connelly MG, Winkelmann RK. Solid facial edema as a complication of acne vulgaris. Arch Dermatol 1985; 121: 87-90

${ }^{5}$ Friedman SJ, Fox BJ, Albert HL. Solid facial edema as a complication of acne vulgaris: Treatment with isotretinoin. J Amer Acad Dermatol 1986; 15: $286-289$

${ }^{6}$ Uhara H, Kawachi S, Saida T. Solid facial edema in a patient with rosacea. J Dermatol 2000; 27: 214-216

${ }^{7}$ Hölzle E, Jansen Th, Plewig G. Morbus Morbihan - Chronisch persistierendes Erythem und Ödem des Gesichtes. Hautarzt 1995; 46: $796-798$

${ }^{8}$ Djavari D. Solides persistierendes Gesichtsödem als seltene Komplikation einer Acne juvenilis. Akt Dermatol 1990; 16: 207-208

${ }^{9}$ Petzold O. Solides persistierendes Gesichtsödem bei lupoider Rosazea. Z Hautkr 2002; 77: 157-158

${ }^{10}$ Krengel S, Kölmel KF. Differentialdiagnostik bei Lidschwellung. Hautarzt 1996; 47: 2 - 19

${ }^{11}$ Jansen Th, Regele D, Schirren CG, Trautner B, Plewig G. Persistierendes Erythem und Ödem des Gesichtes bei Rosazea und Lymphgefäßdysplasie. Hautarzt 1998; 49: 932 - 935

12 Jungfer B, Jansen TH, Przybilla B, Plewig G. Solid persistent facial edema in acne: successful treatment with isotretinoin and ketotifen. Dermatology 1993; 187: $34-37$

${ }^{13}$ Mazzatenta C, Giorgino G, Rubegni P. Solid persistent facial oedema (Morbihan's disease) following rosacea, successfully treated with isotretinoin and ketotifen. Br J Dermatol 1997; 137: 1020 - 1021

${ }^{14}$ Helander I, Aho HJ. Solid facial edema as a complication of acne vulgaris: treatment with isotretinoin and clofazimine. Acta Derm Venereol Stockh 1987; 67: 535-537 